\title{
Venenos de flecha de Colombia
}

\author{
Kalman Mezey
}

Rev. Acad. Colomb. Cienc. Ex. Fis. Nat. 1947, 7 (27): 319-323.

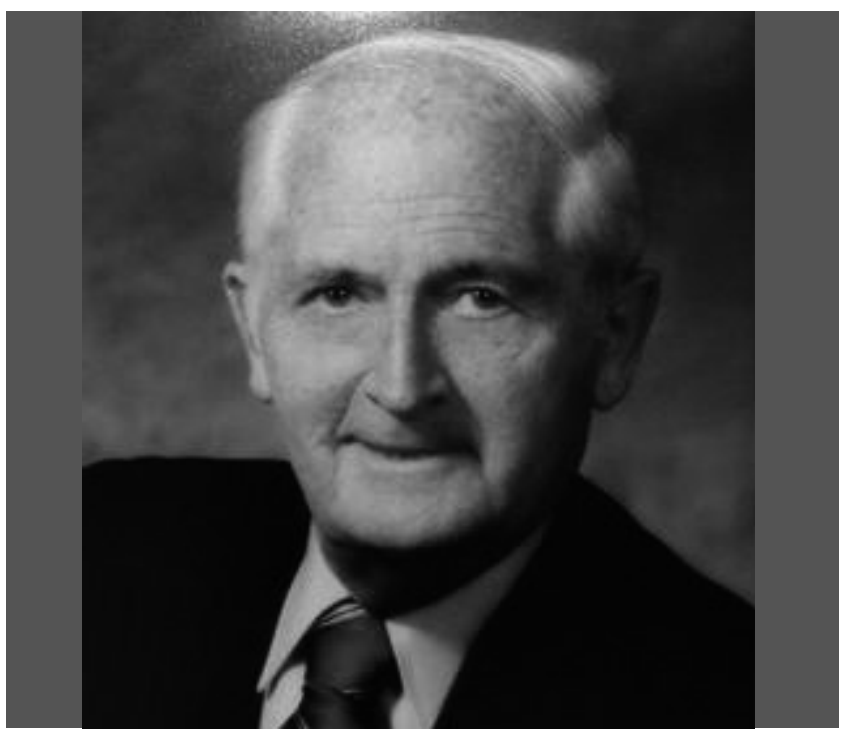

\section{Kalman Mezey (1909-2007)}

El doctor Mezey nació en Nagyvárad (Hungría); estudió medicina en la Universidad de Basilea, especializándose en Medicina Interna y Farmacología, allí y en Viena. Fue profesor desde 1941 de la Facultad de Medicina de la Pontificia Universidad Javeriana en Bogotá, así como del Departamento de Farmacia de la Facultad de Medicina Veterinaria de la Universidad Nacional de Colombia.

Se interesó por los efectos farmacológicos de plantas colombianas en humanos y animales, especialmente por sus efectos cardiovasculares, diuréticos y alucinogénicos, entre otros. Plantas como digital (Digitalis purpurea), Berberis spp., bejuco blanco (Vitis tiliifolia), yopo (Anadenanthera peregrina) y pacurú-niaara (Ogcodeia ternstroemiiflora) fueron de su interés experimental, asi como el exudado de la rana Co-Coi.

Además de su actividad académica, también tuvo vínculos con la industria farmacéutica en Laboratorios CUP (Colombia) y en Merck Sharp \& Dohme (EEUU), donde fue director de investigación clínica. Después de muchos años en Colombia, se vinculó a la New Jersey Medical School (EEUU), en la cual continuó su actividad científica.

Información suplementaria disponible en: http://www. legacy.com/obituaries/nytimes/obituary.aspx?n=kalmanmezey\&pid=97037256
Se describen en el artículo los aspectos farmacodinámicos experimentales de extractos obtenidos de la secreción de la corteza del árbol conocido como pacurú-niaara (árbol venenoso) por los catíos (Ogcodeia ternstroemiiflora, Moraceae), asi como de la piel de una pequeña rana llamada la rana cocoi que posiblemente sea una Phyllobates, utilizadas entonces por algunas tribus del Chocó para impregnar flechas y dardos, destinadas a las cacerías.

El autor analiza los efectos tóxicos de esos extractos en perro, gatos y conejos a dosis extremadamente bajas y detecta fuertes respuestas fisiológicas diferenciales a nivel del sistema nervioso central y del cardiovascular, tales como vómito, diarrea, cambios en la presión sanguínea y bradicardia, entre otras. En cuanto al extracto de la rana, se presentaron otros síntomas como pérdida del equilibrio e hinchazón del abdomen. De todas maneras, se demostró a través de dichos experimentos que el órgano blanco principal era el corazón, aunque éste era un tanto difuso en el caso del venero de rana: no hubo ninguna similitud con el mecanismo de acción del curare.

Estos fueron los primeros intentos para estudiar científicamente nuestra rica biodiversidad-aplicada a la etnomedicina, los cuales, por desgracia, no lograron concretarse en nuestro contexto científico. Además, en el caso de la rana Co-Coi, este artículo ha sido un referente mundial, que favoreció el posterior aislamiento y determinación de la actividad cardiotóxica de los esteroides presentes en dicho veneno, como la batracotoxina.

Si bien esas sustancias como tales no son medicamentos, sus mecanismos de acción permitieron conocer el funcionamiento de la bioquímica celular, a través de los cuales es posible diseñar nuevas drogas. Como bien lo dice el Dr. Mezey "el veneno de ayer es el medicamento del mañana”

Nota: el nombre científico actual de la Moraceae conocida como "pacurú-niaara" o "árbol venenoso" Ogcodeia ternstroemiiflora (Mildbr.) es Naucleopsis terstroemiiflora (Mildbr.) C. C. Berg

Luis Fernando Echeverri, Ph.D. Miembro Correspondiente 


\section{VENENOS DE FLECHA DE COLOMBIA}

(TRABAJO PRESENTADO AL PRIMER CONGRESO INTERAMERICANO DE MEDICINA, RIO DE JANEIRO 1946)

KALMAN MEZEY

Profesor de la Facultad de Medicina de la Universidad Javeriana y Director del Departamento de Investigaciones del Laboratorio cuP.

El veneno de ayer es el medicamento de mañana.

El hombre primitivo en la lucha por su existencia - sea para defenderse de sus agresores 0 bien para conseguir su alimento - acudió a su intelecto para imponerse a la fuerza del ambiente. Posiblemente así nació la primera "arma química" de la historia: el veneno de flecha.

Definimos un veneno como cualquier agente quimico capaz de alterar la integridad anatorio-histológica o el funcionamiento fisiologico de las células, tejidos, órganos o sistemas del ser vivo. Si quisiéramos interpretar la palabra "droga" llegaríamos a la misma definición; sólo que el intento perseguido con las drogas es el de que su actividad se dirija en sentido contrario, es decir: cambiar lo patológico en fisiológico. En efecto, las drogas más poderosas y más eficaces de nuestro arsenal terapéutico actual, por ejemplo la estricnina, el arsénico, la atropina y tantos otros son al mismo tiempo los venenos más temidos. Además, hay un aspecto cuantitativo en la diferenciación entre droga y veneno; así, la aspirina, conocida como una de las drogas más inocuas, obra como tóxico en dosis de quince a veinte veces superiores a las dosis usuales terapéuticas. Por lo tanto, se puede decir que la diferencia esencial entre un veneno y una droga, resulta de la diferencia en la relación cuantitativa que hay entre el efecto y la dosis empleada para obtenerlo.

Los venenos de flecha presentan uno de los casos más instructivos de la penetración del empirismo en la terapéutica. Recordemos que la veratrina, aconitina, estrofantina, ouabaína, estricnina, veneno de serpientes, la rotenona y últimamente el veneno de flecha de mayor mito, el "curare", comenzaron a emplearse como venenos de flecha de los indígenas de los distintos continentes, $\mathrm{y}$ han resultado como drogas indispensables a la terapéutica moderna.

Fue en parte la curiosidad y en parte el interés por la potencia prospectiva de los venenos de flecha, el motivo para que nuestro programa de investigaciones experimentales hiciera ocupar a estas substancias un papel preponderante: dar una orientación sucinta sobre los trabajos relacionados con el primer análisis farmacodinámico de algunos venenos de flecha usados en suelo colombiano. Este es el objeto de la presente comunicacion. No aspiramos a presentar un relato completo, ni puede darse por conocida la extensa y aún compleja serie de productos usados por los indígenas; tan solo nos referiremos a trabajos personales adelantados hasta la fecha.

\section{"PACURU NIAARA"}

Fue C. Uribe Piedrahita quien en 1920 (1) hizo la primera mención científica de un veneno usado por los indígenas de la tribu de los Catíos de la región de Urabá, Departamento de Antioquia, Colombia, para envenenar los dardos de sus bodoqueras o cerbatanas. C. G. Santesson (2), ignorando la publicación de Uribe describió los efectos principales del veneno y concluyó que posiblemente se trataba de una sustancia cardio-activa. Sin embargo, debido quizás a la escasez de material de que dispuso este autor, no se logró un análisis farmacodinámico del veneno.

En estrecha colaboración (3) con C. Uribe Piedrahita y a base de una larga serie de experimentaciones hechas en el Departamento de Investigaciones Experimentales del Laboratorio CUP de Bogotá, con el jugo virgen del árbol Pacurú Niaara recolectado por C. Uribe, hemos logrado determinar el mecanismo de acción de este veneno en el organismo animal.

El veneno se saca de la corteza de un árbol de la familia de las Artocarpóideas, posiblemente, según C. V. Mortton, de la especie Ogcodeia ternstroemiiflora. El jugo se obtiene por incisiones hechas en la corteza en forma espiral, desde la base del tronco hasta arriba; los indigenas lo reciben en un tubo de bambú o en una calabaza pequeña, y luego lo acercan al fuego hasta que el veneno obtiene un color moreno $\mathrm{y}$ en este estado lo untan a los dardos.

En los ensayos de toxicidad general hemos visto que la dosis mínima tóxica del jugo virgen es efectiva en perros y por la vía intramuscular, de 0,015 cc. por kg. de peso. El veneno se absorbe con gran rapidez por el tejido muscular. Los efectos más característicos de la intoxicación son: a) vómitos repetidos a intervalos de 5 a 10 minutos, los cuales pueden contrarrestarse con barbitúricos; b) diarreas; c) bradicardia, y d) disminución de la frecuencia respiratoria. La dosis mínima letal es de 0.05 cc. por kg. de peso. Las fases finales de la intoxicación se manifiestan por hipodinamia, tambaleo y salivación fuerte. Los conejos y las ratas soportan dosis mayores. Las dosis mínimas letales por la vía oral son 60 veces superiores a las empleadas por vía parenteral, por lo cual suponemos que los jugos digestivos destruyen, o el hígado neutraliza, una gran parte del veneno ingerido.

Los ensayos de farmacodinamia se realizaron en el animal entero (perros, gatos y conejos) y en órganos aislados. En el animal entero el efecto más llamativo del veneno es el gran aumento de la pre- 
sión sanguínea que se produce por dosis de $0.003 \mathrm{cc}$. de jugo por $\mathrm{kg}$. de peso y por vía intravenosa. La presión sanguínea sube en dos a tres minutos después de la inyección a valores de 40 a $60 \mathrm{~mm}$. de Hg. más altos de los que tenía el animal inicialmente. Al mismo tiempo se produce disminución de la frecuencia cardíaca. Estos efectos se mantienen una o dos horas en forma constante.

Dosis un poco más altas, de $0,009 \mathrm{cc} . / \mathrm{kg}$. también producen: primero, un ascenso de la presión, pero al cabo de cinco a ocho minutos bruscamente vienen oscilaciones muy grandes, bradicardia fuerte e irregularidad del ritmo; algo semejante a "delirium cordis" que termina con la caída brusca de la presión y muerte del animal. Dosis aún más altas, de 0,1 cc. $/ \mathrm{kg}$., producen un cuadro más dramático: ascenso brusco y fuerte de la presión, paro respiratorio y caida vertical de la presión sanguínea, todo esto dentro de dos a tres minutos después de la introducción intravenosa del veneno. La segunda fase de la intoxicación recuerda el cuadro de intoxicación que producen los glucósidos cardioactivos de la digital, el estrofanto, la ouabaina etc. Hemos hecho unos ensayos comparativos y en las mismas condiciones de experimentación. Las gráficas números $2,3,4$ y 5 demuestran en forma clara la semejanza de la acción de la estrofantina, la ouabaina $\mathrm{y}$ de un extracto inyectable de la Digitalis purpúrea de Bogotá (Folidigal CUP) con el efecto de un extracto purificado de Niaara.

En el caso del Niaara posiblemente se trata de un cuerpo cardiotónico con acción muy semejante a la de los cuerpos medicamentosos más potentes en la terapéutica de la debilidad muscular cardíaca. Esta suposición se ha reforzado por el análisis más detenido del mecanismo del efecto hipertensor, con el resultado de que en la verificación del efecto del Niaara, éste no se debe a participación del sistema nervioso vegetativo, ya que no se altera ni por atropinación ni por ergotaminación previa del animal. Tampoco se observó alteración del efecto después de la desconexión de las zonas quimio y presorreceptoras del seno carotídeo. Igualmente se produjo el efecto hipertensor en el animal mantenido con respiración artifical, por lo que sospechamos que se trata de un efecto directo sobre el músculo cardíaco. Este efecto se comprobó por ensayos hechos en el corazón aislado de ranas y de sapos, obteniendo una disminución de la freeuencia y un aumento de la amplitud de las contracciones cardíacas (gráfica $N^{0}$ 6).

En la primera fase de su acción este veneno obra sobre la respiración por disminución de la frecuencia. El efecto corresponde cronológicamente a la fase de ascenso de la presión sanguínea, y puesto que se produce también después de la desconexión de ambos senos carotídeos, se debe, o al aumento de la oxigenacion y mayor transporte de $\mathrm{CO}_{2}, 0$ a un efecto directo sobre el centro respiratorio. Esta última posibilidad se descarta por el hecho de que la frecuencia respiratoria vuelve en la segunda fase de la intoxicación a su estado original.
De manera que nos inclinamos a suponer que la hipopnea se debe más bien al contenido de $\mathrm{CO}_{2}$, disminuído en el centro respiratorio.

En el intestino aislado el jugo del Niaara obra aumentando el tono y la amplitud de las contracciones peristálticas. En el útero aislado este veneno produce una contracción violenta, una tetanización muy semejante a la que producen los alergenos en el útero de la cobaya sensibilizada. La tetanizací́n producida por el Niaara cede solamente después de la eliminación del veneno por varios lavados de la solución en que se suspende la preparación. Tanto en el intestino como en el útero se trata posiblemente de acciones directas del veneno sobre los elementos musculares lisos.

En cuanto a las acciones del veneno sobre los elementos nerviosos motores periféricos y la musculatura estriada, según ensayos hechos en la preparación nervo-muscular, el Niaara no tiene efectos semejantes a los que produce el Curare, por no alterar la excitabilidad indirecta de la musculatura esqueletal. Tampoco hay alteración de la conductibilidad de los nervios motores. La excitabilidad directa de los músculos no es influída por el Niaara.

\section{"USCHARIN"}

El "uscharin" (viene del árabe uschar) es uno de los glucósidos cardioactivos de la planta Calotropis procera (India, Arabia, Persia) y Calotropis gigantea (India, Islas Malayas). El Calotropis porcera se ha usado y se usa todavía en el Sudán como veneno de flechas. Ocasionalmente tuvimos conocimeinto de la existencia de grandes cantidades de Calotropis procera en la Costa Atlántica y sin poder precisar si esta planta fue usada en la preparacion de venenos de flechas, no vacilamos en someter su jugo lechoso a una investigación farmacodinámica. Estos ensayos así como el aislamiento químico de sus principios activos, están en marcha. Por ahora daremos cuenta de los fenómenos de intoxicación y de los efectos principales del veneno.

ToxicidaD: En el conejo, la inyección intravenosa de 0,1 cc. jugo $/ \mathrm{kg}$. produce bradicardia intensa, arritmia y dispnea. El animal intoxicado se sostiene en pie pero no puede levantar o sostener la cabeza, la cual se fleja entre las extremidades anteriores. De vez en cuando el animal sufre una débil convulsión y alcanza a levantar la cabeza, pero luego la deja caer después de pocos segundos. Observamos también hipo, $y$, como principio de náuseas, se observaron bostezos. El animal continúa así durante 40 a 60 minutos, tiempo en el cual los síntomas empiezan a regresar. El cuadro de la intoxicación recuerda mucho a la intoxicación digitálica especialmente por la bradicardia y la debilidad de los músculos de la nuca, que según C. C. Santesson y Strindberg (4) parece ser característica de la intoxicación digitálica.

En los ensayos farmacodinámicos se ha visto que dosis de 0.09 cc./kg. jugo por vía intravenosa, pri- 


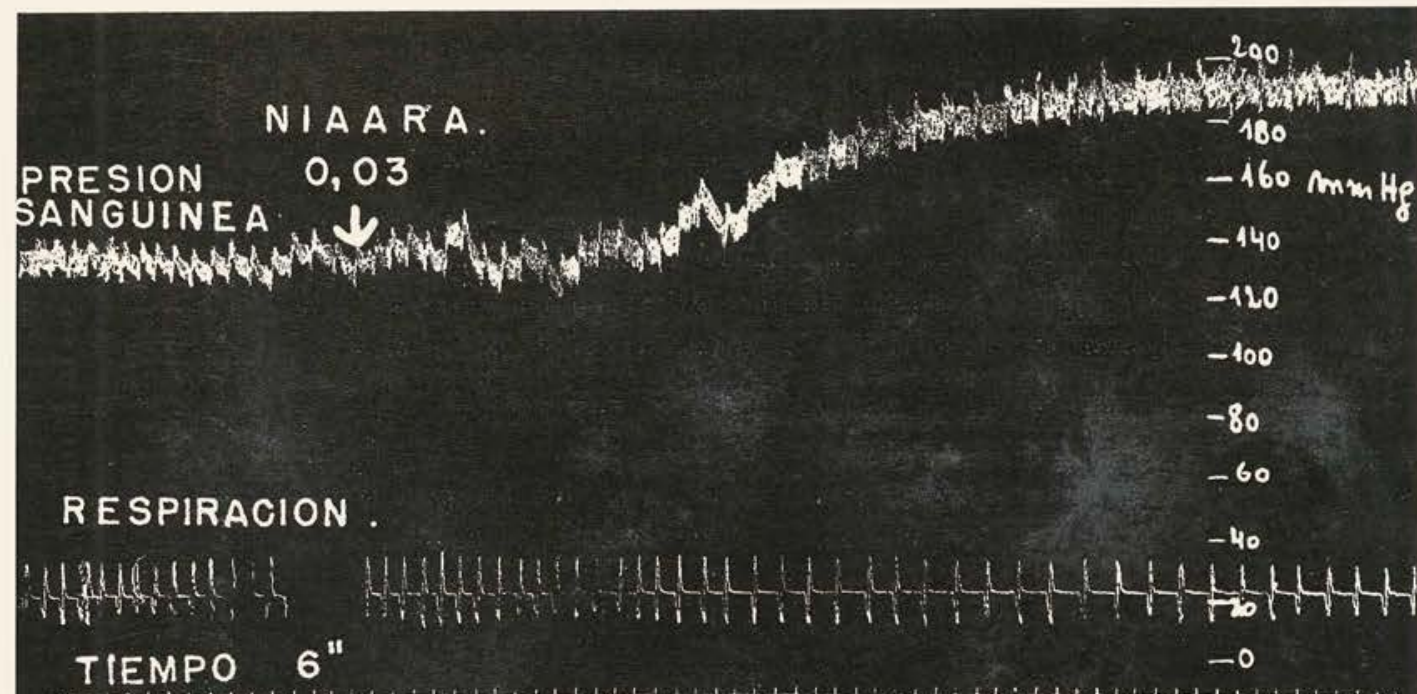

Gráfica No 2. - Efecto de un extracto purificado del Niaara sobre la presión sanguínea y la respiración.

NIAARA 3 G.

SIN FRACCION ALCALOIDICA.

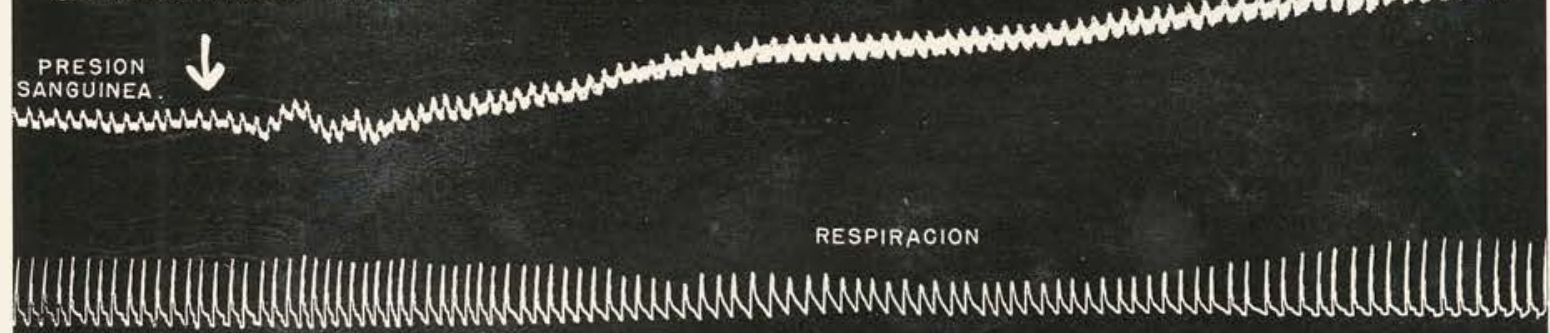

TIEMPO $6^{\text {" }}$

Gráfica No 3. - Efecto de la Estrofantina sobre la presión sanguínea y la respiración en un perro de 7 kg.

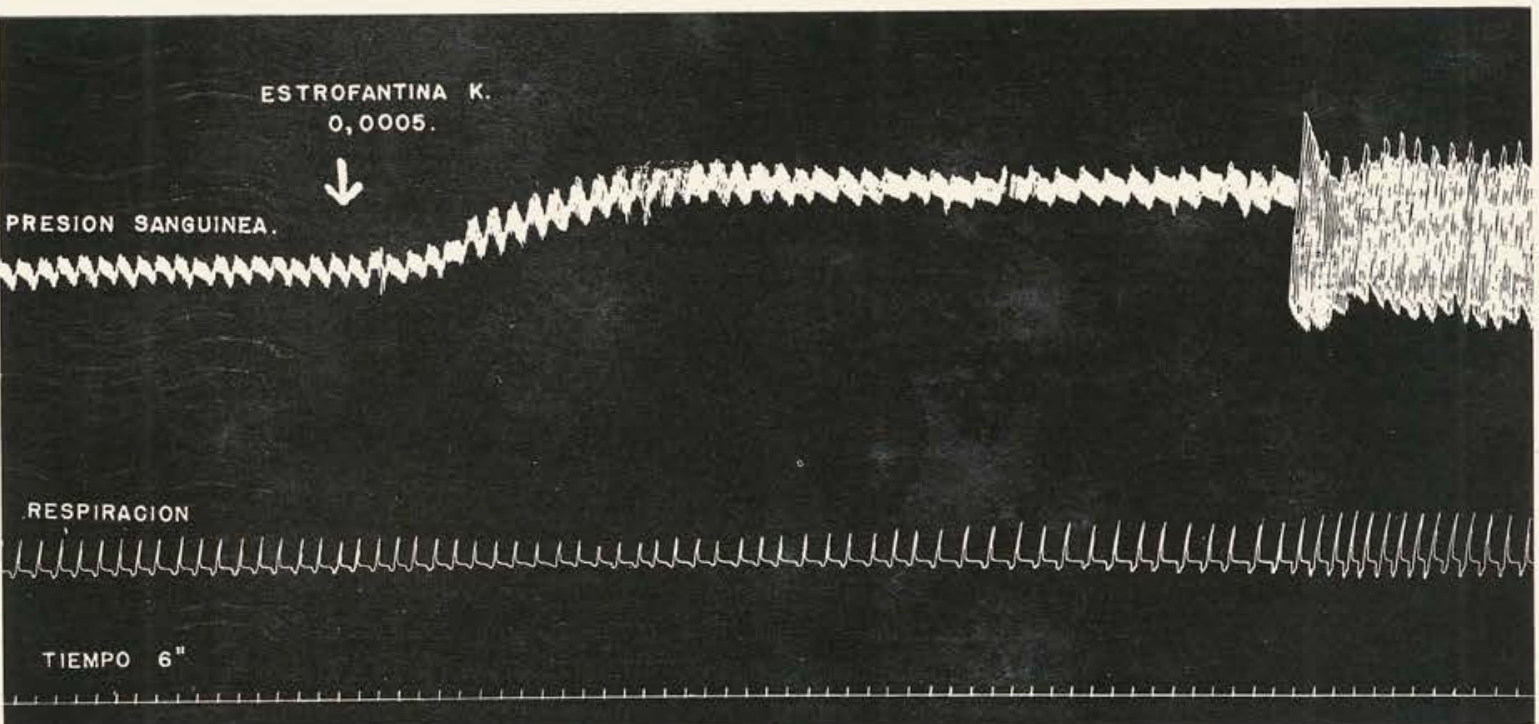




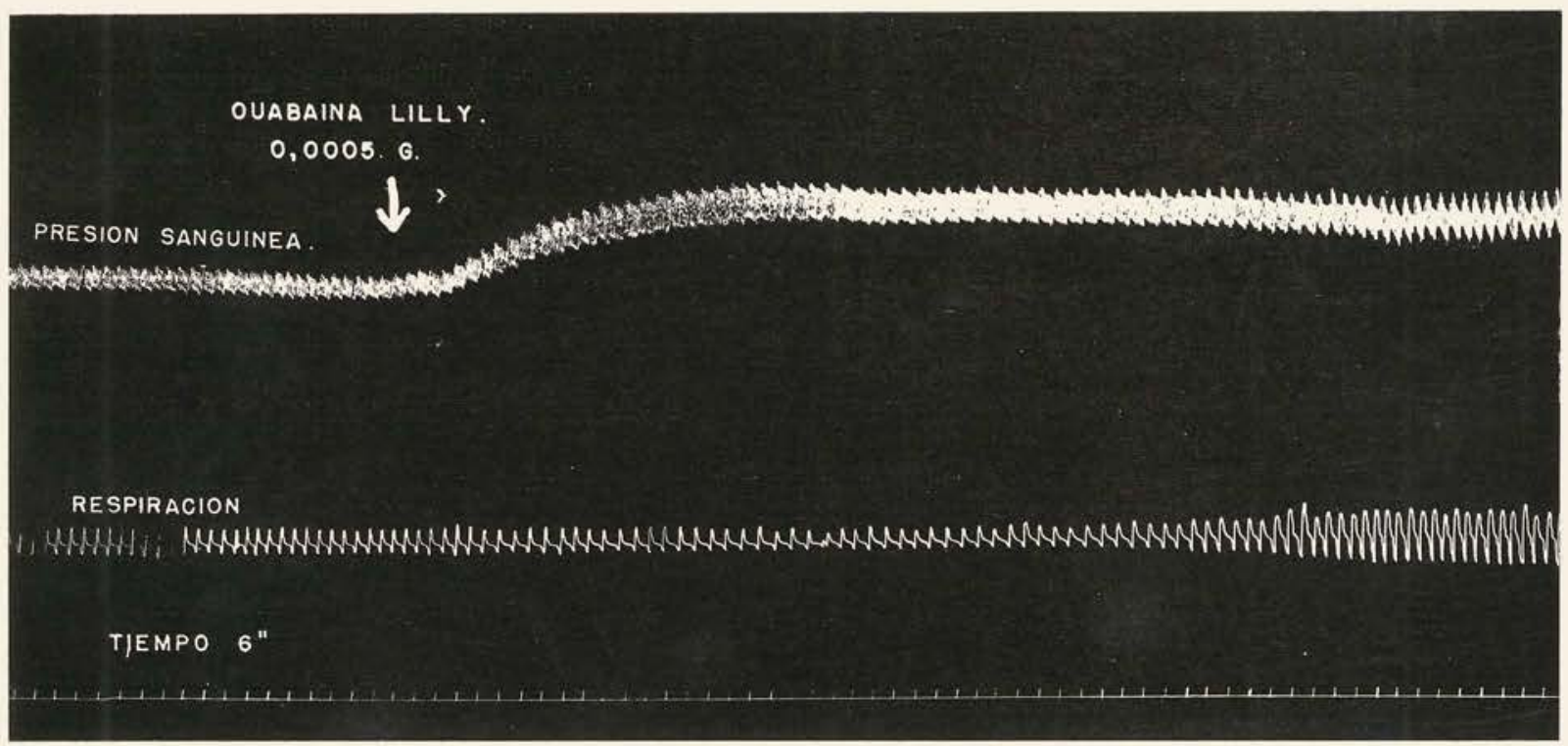

Gráfica No 5. - Efecto del Folidigal (0.2 gramos de hoja seca de Digitalis purpurea de Bogotá) sobre la presión sanguínea de un perro de $9.5 \mathrm{~kg}$.

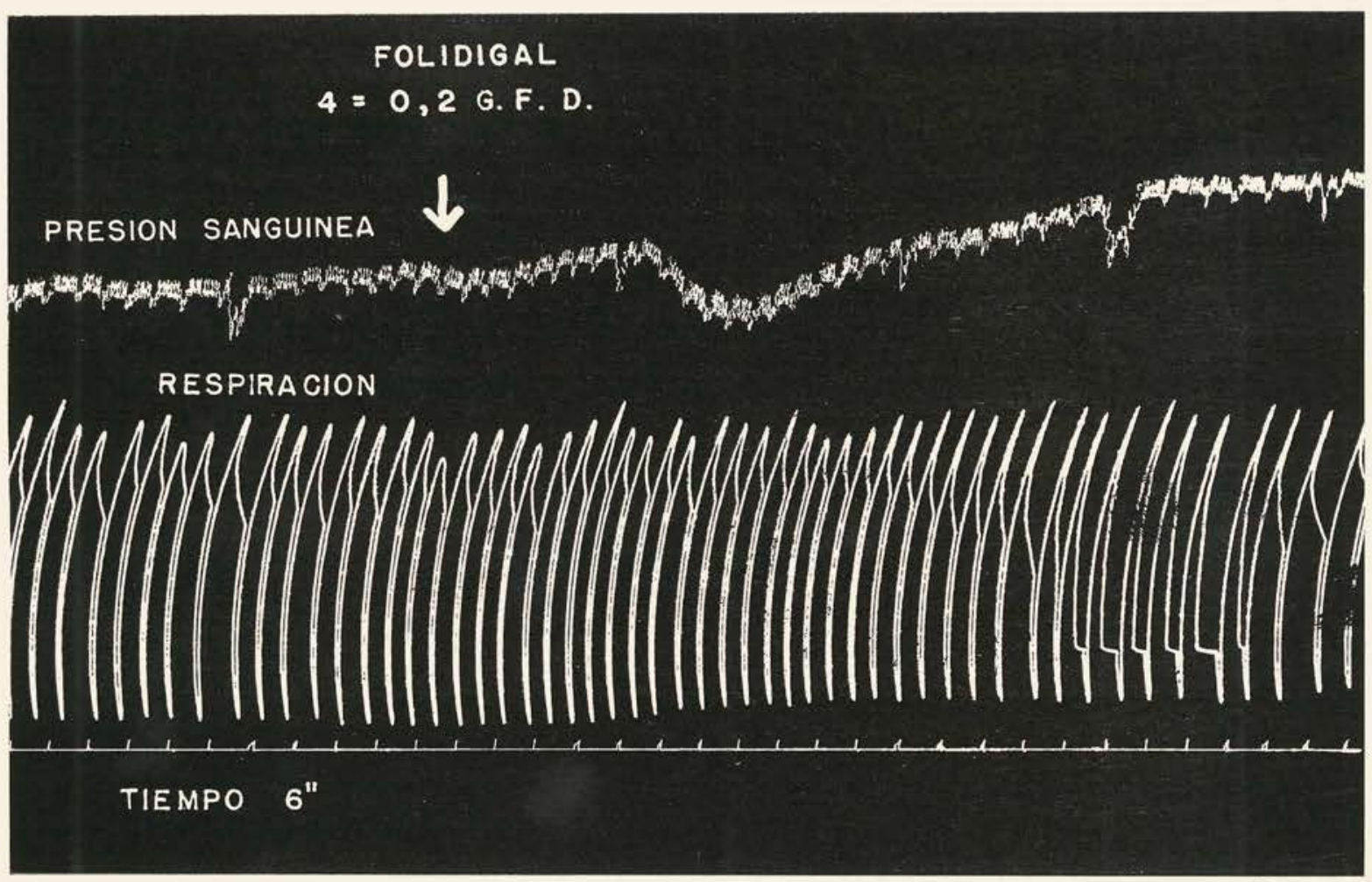

Gráfica N9 6. - Efecto del Niaara 1:7500 en el corazón aislado.

\section{NI A A R A$$
1: 7500
$$ \\ $\downarrow$}

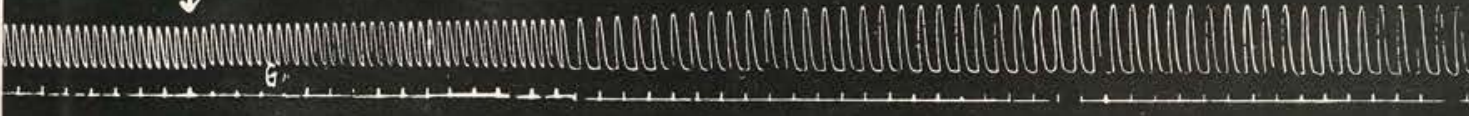


mero aumenta considerablemente la presión san. guínea (gráfica No 7) que alcanza a los cuatro minutos después de la inyección o valores de 40 a $50 \mathrm{~mm}$. de $\mathrm{Hg}$. mayores que la inicial. Luego, como en el caso de los glucósidos cardioactivos, aparecen pulsaciones arritmicas de gran amplitud y de baja frecuencia. La respiración se altera en la primera fase de la acción, es decir durante el ascenso de la presión, haciendo disminuír la frecuencia y la amplitud, mientras que desde el momento de la aparición de las "pulsaciones tipo vagoirritación" hay más bien hiperpnea. En cuanto al mecanismo de estas alteraciones de la respiración nos referimos a las consideraciones hechas en el caso del Niaara.

En los órganos aislados se not6 aumento del tono y del peristaltismo de aquellos de musculatura lisa.

Planta
Digitalis purpurea y lanata
Strophanthus hispidus y gratus
Adonis vernalis
Convallaria maialis
Acocanthera Ouabaio, Deflersi, etc.
Nerium Oleandri
Thevetia nerilfolia
Adenium Boehmianum
Eritrophleum guinense
Helleborus niger
Antiaris toxicaria
Periploca graeca
Scilla maritima
OGCODEIA TERNSTROEMIIFLORA
CALOTROPIS PROCERA

En el corazón aislado del sapo el "uscharin" produce disminnción de la frecuencia y paro diastólico a veces mesosistólico. En la preparación nervomuscular se ha podido demostrar que el veneno no posee acciones curarizantes.

El interés de los pocos datos que acabamos de exponer sobre el "uscharin" reside en el hecho de haber analizado por la primera vez, el jugo lechoso de una planta colombiana (en la Costa Atlántica de Colombia se la llama "Algodón de Seda") que en otros lugares del mundo se usa como veneno de flecha $y$ que contiene un principio cardioactivo.

Se agregan así dos venenos: el "Pacurú Niaara" y el "Algodón de Seda" a otras plantas cardioactivas, las cuales son:

\begin{tabular}{|c|c|}
\hline Lugar & Principlos activos \\
\hline Europa & Glucósidos \\
\hline Africa del Este & Glucósidos \\
\hline Europa, Asia & Glucósidos \\
\hline Asia, Europa & Glucósidos \\
\hline Africa del Este & Glucósidos \\
\hline Asia, Europa & Glucósidos \\
\hline México & Glucósidos \\
\hline Africa del Sud & Glucósidos (Echujina) \\
\hline Africa Central & Alcaloide (Eritrofleina) \\
\hline Europa & Glucósidos y Alcaloides \\
\hline Java, India & Glucósidos \\
\hline Mar Negro & Glucósidos \\
\hline Europa & Glucósidos \\
\hline Colombia & Glucósidos \\
\hline Colombia & Glucósiđos \\
\hline
\end{tabular}

\section{VENENO DE LA RANA DENDROBATES (Co-Coi)}

Las tribus indígenas de los Noanamas, Cunas y Chocoes, habitantes del Departamento de Antioquia y la Intendencia del Chocó en la República de Colombia, usan desde hace siglos como veneno de flecha la secreción lechosa de las glándulas dérmicas de un batracio perteneciente al género Dendrobates. La primera descripción del veneno, del modo de prepararlo y datos sobre su acción, se deben a A. Posada Arango (4). Este autor, a base de unos pocos ensayos y con maravilloso criterio experimental para su época, concluyó que el veneno mata por parálisis del sistema nervioso central, ya que mientras la contractibilidad muscular queda inalterable, la irritabilidad eléctrica indirecta se inhibe. En la autopsia inmediata de los animales intoxicados, el corazon se encontró dilatado y lleno de sangre. Santesson (5) que examinó la acción tóxica de este veneno en ranas, cree que obra primero por parálisis muscular y sólo en la segunda fase de su acción letal hay parálisis del sistema nervioso central y paro cardíaco en sístole. Hay dos contradicciones en las observaciones de los autores mencionados: mientras que A. Posada Arango habla de la conservación de la contractibilidad muscular y de un corazón lleno de sangre (por lo tanto un paro diast6́lico); Santesson observó parálisis muscular y paro cardíaco sistólico. En vista de que, según nuestros conocimientos, no hay un estudio completo farmacodinámico de este veneno, creemos de interés resumir aquí nuestras investigaciones sobre el particular:

Para los ensayos hemos usado virotes o dardos envenenados desde hace 15 años; la cantidad aproximada que lleva cada virote es alrededor de dos miligramos.

La dosis minima letal del veneno estudiado en perros y conejos es, por vía intramuscular o intravenosa, alrededor de 0.0001 gmo. por $\mathrm{kg}$. de peso. La absorción del veneno por el tejido muscular es muy rápida, puesto que los primeros síntomas de la intoxicación se presentan dos a cuatro minutos después de la inyección. Dosis diez veces superiores a las minimas letales parenterales, se soportan por la vía oral sin causar trastorno alguno. Introduciendo las dosis letales en la vena mesentérica tampoco se producen fenómenos tóxicos. Resulta, pues, que el hígado posiblemente destruye el veneno; sin embargo, en ensayos "in vitro" no se pudo verificar tal destrucción por el tejido hepático. La sangre de los animales letalmente intoxicados no presentó hemolisis.

Los sintomas más llamativos de la intoxicación son: dispnea, bradicardia y pérdida del equilibrio. En el curso de la intoxicación el animal pierde el equilibrio y cae; el tren posterior parece paralizado. Si en este estado se inyecta estricnina (sul- 
fato de estricnina) $0,0003 \mathrm{~g} / \mathrm{kg}$. el animal reaccio na $y$ muestra convulsiones.

En el análisis farmacodinámico de la acción del veneno aplicado por vía intramuscular o intravenosa domina la baja de la presión sanguínea acompañada de inhibición respiratoria (gráfica $N^{\circ} 8$ ).

La dosis mínima activa, capaz de producir tales reacciones es alrededor de $0,00008 \mathrm{gmo} . / \mathrm{kg}$. ¿Qué relación existe entre los dos efectos? ¿Se paraliza primero la respiración y la disminución de la presión se debe sólo a la anoxemia, o hay parálisis simultánea del centro respiratorio y vasomotor, o hay daño cardíaco directo muscular? A base de varios ensayos hechos en animales previamente tratados con atropina (desconexión farmacodinámica del vago) y en otros mantenidos con respiración artificial, creemos poder concluír que el efecto inmediato letal en dosis grandes del veneno, se debe a una parálisis respiratoria. En el curso de la intoxicación el veneno afecta también al músculo cardíaco porque la muerte, aunque se retarda un poco, siempre se presenta aún en el animal mantenido con respiración artifical. Es muy significativo que en el curso de la intoxicación no letal, la frecuencia respiratoria se restablece en diez minutos después de una disminución transitoria, mientras que la hipotensión fuerte es duradera; de modo que bien puede tratarse de un debilitamiento del músculo cardíaco. Se refuerza tal sospecha por los resultados obtenidos en el corazón aislado del sapo, puesto que el veneno, en la dosis mínima activa que es alrededor de $1: 200.000$, resultó como un inhibidor cardíaco produciendo disminución de la frecuencia y de la amplitud de las contracciones cardiacas. La concentración del veneno que resultó como inhibidor cardíaco, es demasiado grande en relación con la dosis mínima eficaz en el perro. Sin embargo, hay que tener en cuenta la poca sensibilidad de los sapos a drogas que obran sobre el corazón. Además, parece que el veneno de Dendrobates no afecta a los sapos, puesto que la inyección de 0.0003 gmos. del veneno en el saco linfático de un sapo de 85 gmos., o sea 0.0035 gmo. $/ \mathrm{kg}$., es decir 35 veces más que la dosis mínima letal en el perro, no produjo efecto tóxico alguno. Quedamos así en cierta contradicción con E. R. Dunn (6), quien coleccionando ranas diversas las colocó con uno o más Dendrobates en un saco de tela liviana y al poco tiempo todas las ranas, con la única excepción de los Dendrobates, habian muerto, hecho del cual concluye Dunn que la exudación cutánea de estas ranas había matado a las otras.

En el intestino delgado aislado del conejo se observó aumento del peristaltismo con concentraciones de 1:330.000, mientras que en útero aislado no se observó efecto alguno.

En la preparación nervo-muscular del sapo como en el animal entero (perro) letalmente intoxicado, no hubo alteración de la excitabilidad directa o indirecta de la musculatura esqueletal, de manera que la parálisis muscular aparente que presentaron los animales letalmente intoxicados, se debe, probablemente, más bien a la disminución del volumen circulatorio por la hipotensión arterial.

\section{RYANIA}

Ryania dentata var. tóxica (Dugand) (7)

Humboldt y Bonpland (8) mencionan bajo el nombre de Patrisia affinis una planta cuyas raíces tenían fama, entre los indígenas del Orinoco, de contener un veneno muy poderoso. Santiago Cortés (9) deseribió otra especie, la Ryania tomentosa, llamada vulgarmente "mata cucaracha" como un arbusto muy venenoso con efectos semejantes a los de la estricnina. Gilg (según Engler y Prantl (10), distingue diez especies de este género tóxico que crecen en el norte del Brasil, Guayanas y Colombia. Gleason (11) menciona que la corteza de la Ryania sauricida se usa por los indígenas de la Amazonia brasileña para envenenar caimanes. Y últimamente Nakarai (12), en el Brasil logró aislar un glucósido muy tóxico de la Ryania acuminata.

Consideramos que la Ryania dentata de Orocué, Orinoquia colombiana, merece ser mencionada entre los venenos de flecha, (aún sin tener la certeza de que haya sido usada como tal) por las siguientes razones: la región en donde se encuentra la planta está muy cerca a las regiones en donde se usaban y se usan aún los venenos de flecha $y$ hay seguridad absoluta de que los indígenas conocen las propiedades venenosas de esta planta. Sabiendo que los venenos de flecha muy rara vez se preparan de una sola planta y más bien se obtienen de varias conocidas como venenosas, y teniendo en cuenta que distintos viajeros, desde Sir Walter Raleigh (1595), describieron los efectos del envenenamiento con "curare", observando sintomatologías muy diferentes y hasta contradictorias, debidas seguramente al hecho de que las mezclas de "curare", no son uniformes, porque a veces contienen 17 hasta 28 plantas (Krukoff y Moldenke (13). No nos extrañaría que en las distintas mezclas entrara también la planta, cuyos efectos tóxicos y farmacodinámicos resumiremos en lo siguiente:

Después de habernos convencido por ensayos hechos con los extractos hidroalcohólicos de que en el caso de la especie estudiada, en realidad se trataba de una planta tóxica (dosis mínima letal por vía intramuscular y por kgmo. de peso: extracto hidroalcohólico de 0.1 gmo. de hoja seca) intentamos el aislamiento del principio activo de la planta. Hemos logrado aislar de las hojas un glucósido que se cristalizó en forma de agujas delgadas y cuyas acciones en el organismo animal fueron idénticas a las observadas con los extractos hidroalcohólicos, tratándose por lo tanto del principio activo de la planta.

Toxrcipad: La dosis mínima toxica del glucósido "ryanina" en el conejo, es alrededor de 0,000015 gmos. por $\mathrm{kg}$. de peso por vía intravenosa. Tres minutos después de la inyección el animal presenta primero hiperpnea transitoria, lnego movimien- 


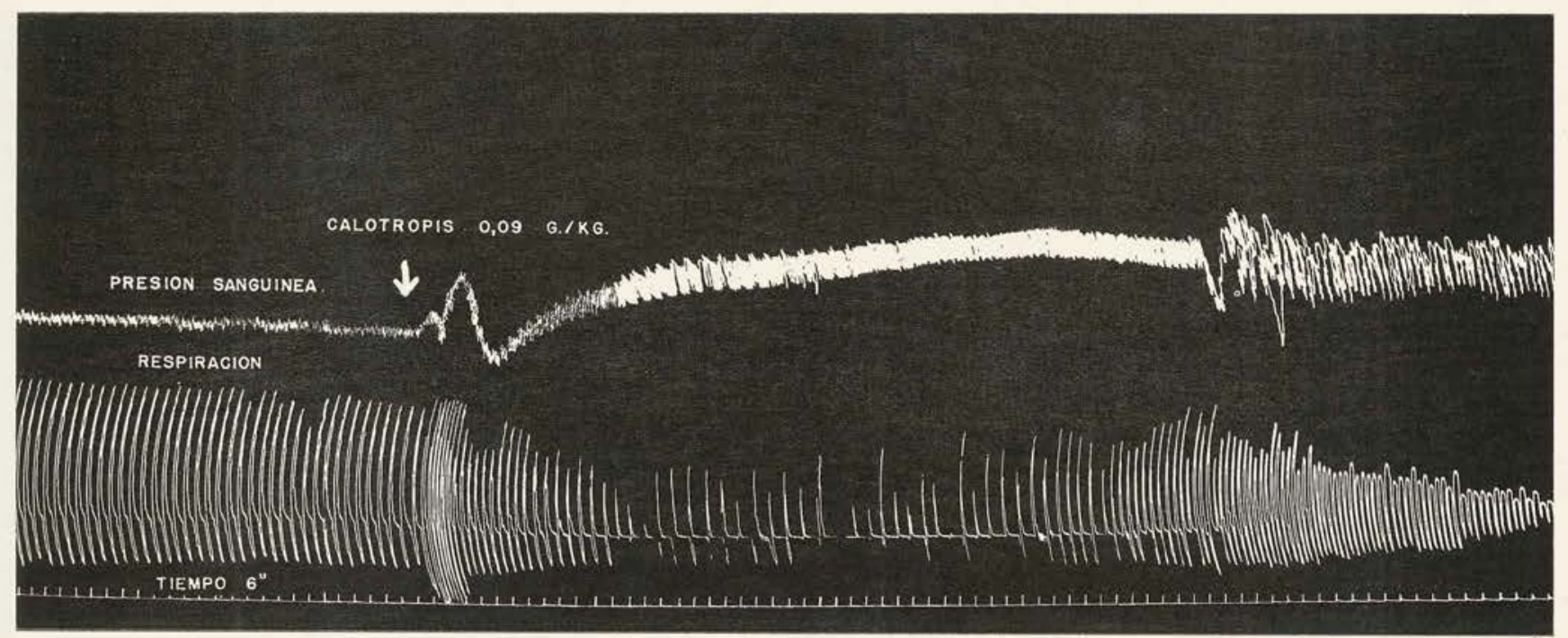

Gráfica No 8. - Efecto del veneno de la rana "Dendrobates chocoensis" sobre la presión sanguínea y la respiración de un perro de $11 \mathrm{~kg}$. Dosis: $0.0012 \mathrm{~g} / \mathrm{kg}$.

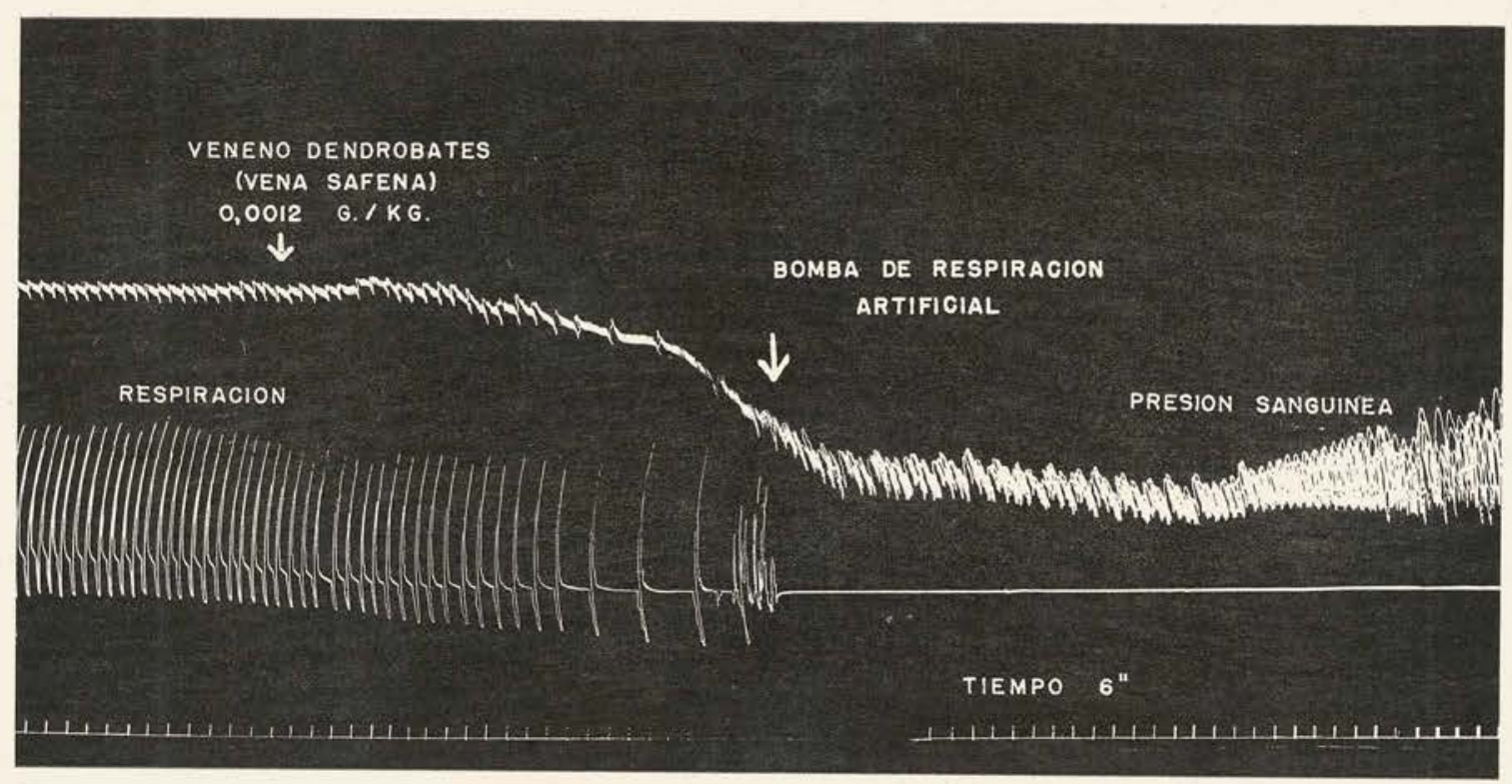

Gráfica No 9. - Efecto de la Ryannia 0.00035 g./ kg. sobre la presión sanguínea y la respiración de un perro de 12 kg.

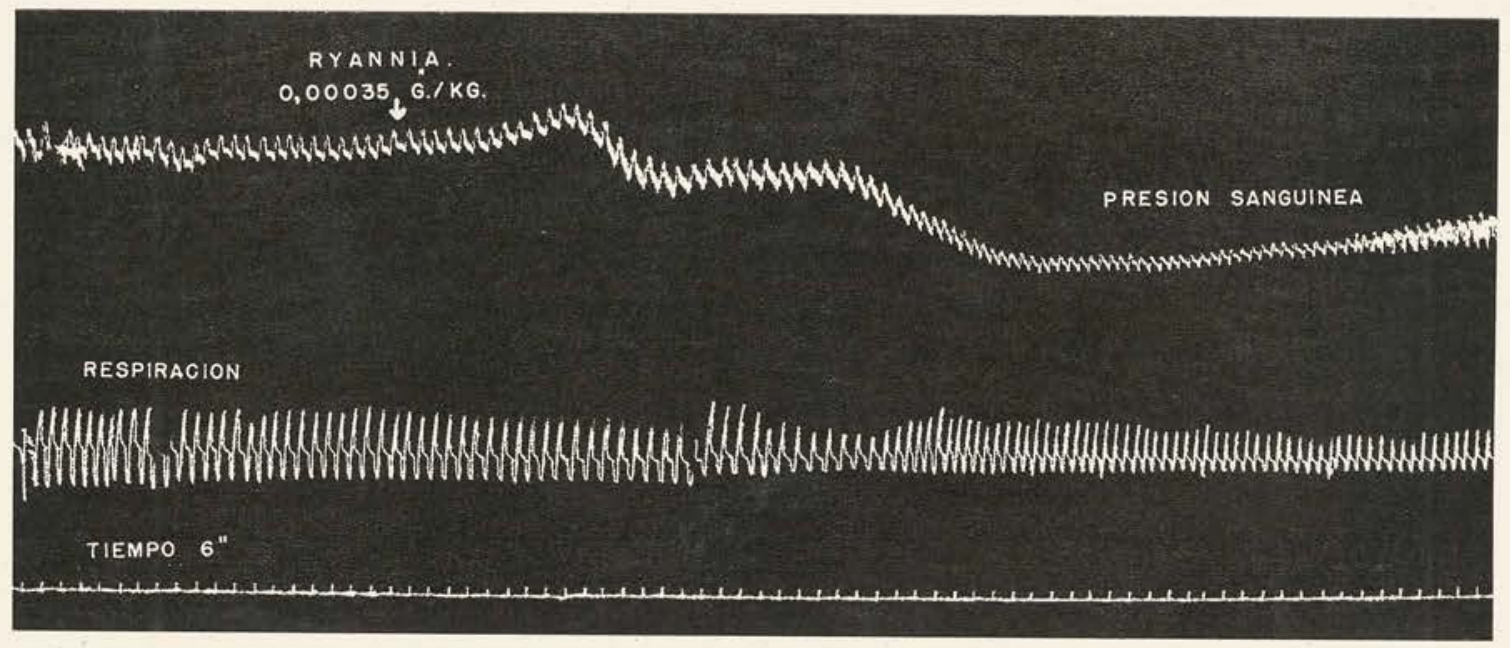


tos inciertos, atáxicos, la cabeza rígida levantada, se voltea de un lado a otro; hay tambaleo y rigidez tan exagerada de la musculatura de las extremidades, que la marcha adquiere el carácter grotesco de "marcha en zancos"; hay midriasis, a veces nistagmus, bradicardia, e hinchazón del abdomen; diez a veinte mnutos después de la inyección el animal muere por paro respiratorio, presentando antes unas convulsiones asfigticas premortales. $\mathrm{Al}$ abrir la caja toráxica, inmediatamente después del paro respiratorio, el corazón se encontró todavía latiendo. Los animales letalmente intoxicados sobreviven un tiempo más largo haciéndoles respira. ción artificial.

Por vía oral, la dosis mínima letal en conejos es diez veces mayor que por la vía parenteral. Se debe esta diferencia posiblemente a la destrucción parcial del glucósido por la acción hidrolítica de los jugos digestivos.

En los ensayos de farmacodinamia en el animal entero, se observó, a consecuencia de la inyección intravenosa de 0.00015 a 0.00035 gmos. de "ryanina" por kgmo. de peso, una alza inicial (gráfica número 9) transitoria (duración 20 a 25 segundos) y luego una disminución duradera de la presión sanguínea. Dosis mínimas parecen destruírse o neutralizarse poco a poco en el organismo animal, puesto que al cabo de una o dos horas la presión puede volver a su valor inicial (gráfica $N^{\circ} 9$ ). En cambio dosis letales producen una hipotensión que se mantiene durante horas. Estas dosis $(0.00015$ a $0.00035 \mathrm{gmo} . / \mathrm{kg}$.) no influyen visiblemente en la respiración; sin embargo, la muerte del animal se produce por paro respiratorio brusco.

Hemos podido aclarar el mecanismo del efecto hipotensor del veneno; posiblemente se trata de una acción paralizante sobre el centro vasomotor, puesto que en el corazón aislado del sapo, no se ha visto efecto depresor con concentraciones muy superiores a las que matan el animal entero. Además, recordamos que en el animal letalmente intoxicado, el corazón sigue uno a tres minutos latiendo después de la suspensión total de la respi- ración. También puede tratarse de un efecto paralizante sobre la musculatura lisa de los vasos, puesto que el veneno disminuye el tono y el peristaltismo de los órganos de musculatura lisa, hecho que hemos comprobado en el intestino y útero aislados. Finalmente, se ha podido averiguar por la misma técnica de experimentación, que en el caso de los venenos anteriormente tratados la "ryanina" no tiene efectos curarizantes.

A pesar de que la terapéutica moderna se orienta cada día más hacia los cuerpos químicos sintéticos, la mayoría de ellos se elabora a base del conocimiento de la acción y de las estructuras químicas de las fuentes naturales, es decir, de las drogas de origen vegetal. Al someter al Primer Congreso Interamericano de Medicina el presente trabajo, deseamos hacer un llamamiento fervoroso de colaboración, a fin de seguir desentrañando los misterios que encierra la vida vegetal $\mathrm{y}$ hacerlos accesibles a la investigación sistemática.

B I B L I O GR A F I A

(1) C. Uribe Piedrahita. - Apuntaciones sobre la geografía médica de la región de Urabá. Tesis, Medellín, 1920.

(2) C. G. Santesson. - Skand. Arch. Physiol. Vol. 55, pág. $230-257,1929$.

(3) C. Uribe P. y K. Mezey. - Revista de la Universidad Nacional de Colombia, Vol. 3, págs. 177-254, 1945

(4) A. Posada Arango. - Le poison de la Rainette des sauvages du Choco, Arch. Méd. Nav., 1871, XVI, pg. 203-213. Traducción original de la Biblioteca de la Facultad de Medicina de Medellín y cit. por M. Phisalix, Animaux venimeux et venins, Masson, 1922.

(5) C. G. Santesson. - Ethnological Studies, Vol. I, página 105, 1935. (Mierofilm).

(6) E. R. Dunn. - Caldasia, Vol. II, No 10, pg. 519, 1944.

(7) Dugand. A. -- Caldasia, Vol. III, No 13, pg. 267, 1945.

(8) Humboldt, A., y Bonpland. - Voyages au Venezuela, 1820

(9) Santiago Cortés. - Flora de Colombia, pg. 113, 1.

(10) Engler y Prantl. - Die Natürlichen Pflanzenfamilien, Vol. 21, pg. 450, 1925.

(11) H. A. Gleason. - Phytologia, 1, pág. 106, 1934.

(12) Nakarai. - Boletím da Escola de Chimica Industrial "Pará", 1942.

(13) Krukoff, B. A., y Moldenke, H. N. - Brittonia, Vol. 3, No 1, pg. 1-74, 1938. 